\title{
FINITE SUPERSOLVABLE WREATH PRODUCTS
}

JOHN R. DURBIN

1. Introduction. In [1], Baumslag found all nilpotent standard restricted wreath products $A$ wr $B$. It is immediate that $A$ wr $B$ is solvable if and only if $A$ and $B$ are. In this paper we prove the following results.

Theorem. $A$ finite wreath product $A$ wr $B$ is supersolvable if and only if:

(i) $A$ is nilpotent,

(ii) either $B$ is Abelian or $B^{\prime}$ is a (nontrivial) p-group with $A$ a p-group for the same prime $p$, and

(iii) for each prime $q$ dividing $o(A), q \equiv 1(\bmod m)$, where $m$ is the exponent of $B / Q, Q$ being the Sylow q-subgroup of $B$ ( $Q$ is unique by (ii), and may be trivial).

Corollary. If $A$ and $B$ are finite and satisfy conditions (i), (ii), and (iii) above, then any extension of $A$ by $B$ is supersolvable.

2. Notation and definitions. All groups considered are finite and are written multiplicatively. $E$ denotes the trivial group and 1 is used for the identity of all groups. Other notation is standard (as found, for example, in [8]).

Let $A$ and $B$ be nontrivial abstract groups, and let $F=A^{B}$ be the direct sum of copies of $A$ indexed by the set $B$. Explicitly, $F$ is the set of all functions from $B$ into $A$, made into a group by componentwise multiplication. For $f \in F$ and $b \in B$, define $f^{b} \in F$ by $f^{b}(y)=f\left(y b^{-1}\right)$ for all $y \in B$. Then for each $b \in B$, the mapping $f \rightarrow f^{b}$ is an automorphism of $F$, and the group of all such automorphisms is isomorphic to $B$. The (standard restricted) wreath product of $A$ and $B, A$ wr $B$, is defined to be the group generated by $F$ and $B$ with relations $b^{-1} f b=f^{b}$ for all $f \in F, b \in B$. Here $F \triangleleft A$ wr $B$, and $A$ wr $B$ is a splitting extension of $F$ by the group of automorphisms $B$. $W$ will be used throughout to designate $A$ wr $B, F$ (called the base group) will always be as defined above, and $K$ will denote the subgroup of $W$ defined by $K=\left\{f \in F: \Pi_{x \in B} f(x) \in A^{\prime}\right\}$.

We say that a group $G$ is supersolvable if and only if it has an invariant series whose factors are of prime order, that is, a series $G=G_{n}, G_{n-1}, \cdots, G_{0}=E$ with each $G_{i} \triangleleft G$ and each $G_{i+1} / G_{i}$ of prime

Presented to the Society, November 25, 1964; received by the editors December 7, 1964. 
order (also called a supersolvable series). $G$ is nilpotent if and only if it has a series $G=G_{m}, G_{m-1}, \cdots, G_{0}=E$ in which each $G_{i} \triangleleft G$ and each $G_{i+1} / G_{i} \subseteq Z\left(G / G_{i}\right)$. The properties of supersolvable and nilpotent groups needed can be found in [3] or [8]. Certain of these, as well as some results on wreath products, are listed for convenience in the following lemma.

LEMMA 0.

(0.1) $W^{\prime}=K B^{\prime}[6$, Corollary 4.5].

(0.2) If $C$ is a subgroup of $A$ and $D$ a subgroup of $B$, then $C$ wr $D$ can be embedded in $A$ wr $B$ [7].

(0.3) Every extension of $A$ by $B$ can be embedded in $A$ wr $B$ [5], [7].

(0.4) $A$ wr $B$ is nilpotent if and only if $A$ and $B$ are p-groups for the same prime $p[1]$.

(0.5) If $G$ is nilpotent then $G$ is supersolvable.

(0.6) If $G$ is supersolvable then $G^{\prime}$ is nilpotent.

(0.7) Subgroups of nilpotent (supersolvable) groups are nilpotent (supersolvable).

(0.8) $G$ is nilpotent if and only if $H$ a proper subgroup of $G$ implies $H$ a proper subgroup of $N_{G}(H)$.

(0.9) $G$ is nilpotent if and only if it is the direct product of its Sylow subgroups.

\section{The proofs.}

Lemma 1. If $A$ wr $B$ is supersolvable, then $A$ is nilpotent.

Proof. If $A$ is not nilpotent, it contains a proper subgroup $H$ with $N_{A}(H)=H(0.8)$. Then $H_{1}=\{f \in K: f(x) \in H$ for all $x \in B\}$ is a proper subgroup of $K \subseteq W^{\prime}(0.1)$. Suppose $f \in N_{K}\left(H_{1}\right) \backslash H_{1}$. Then there exists $x \in B$ such that $f(x)=a \in A \backslash H$. If we choose $y \in B, y \neq x$, and define $g \in H_{1}$ by $g(x)=h, g(y)=h^{-1}$, and $g(z)=1$ for $z \neq x, y$, where $h$ is any element of $H$, we see that $f^{-1} g f \in H_{1}$, so that $\left(f^{-1} g f\right)(x)=a^{-1} h a \in H$. But since $h$ is arbitrary in $H$ this implies $a \in N_{A}(H) \backslash H$, which is impossible. Therefore $N_{K}\left(H_{1}\right)=H_{1}$, contradicting the nilpotency of $K$ $(0.8)$ and $W^{\prime}(0.7)$ and therefore the supersolvability of $W(0.6)$.

Lemma 2. If $A$ wr $B$ is supersolvable, then $B$ is Abelian or $B^{\prime}$ is a (nontrivial) p-group and $A$ is a p-group for the same prime $p$.

Proof. If the lemma is false, then there are primes $p \mid o\left(B^{\prime}\right)$, $q \mid o(A), p \neq q$. Take $a \in A$ with $o(a)=q$ and $b \in B^{\prime}$ with $o(b)=p$. Choose arbitrary distinct elements $x, y \in B$, and define $f \in F$ by $f(x)=a, f(y)=a^{-1}, f(z)=1$ for $z \neq x, y$. Then $f \in K \subset W^{\prime}, b \in W^{\prime}$, $o(f)=q, o(b)=p$, but $f b \neq b f$. (If $a=a^{-1}$ for all $a \in A$, then $o(B)>2$ and 
$x$ and $y$ can be chosen so that we have $f b \neq b f$.) Therefore $W^{\prime}$ is not nilpotent (0.9) and so $W$ is not supersolvable (0.6).

Lemma 3. $A$ wr $B$ is supersolvable if and only if $A$ is nilpotent and $J_{p}$ wr $B$ is supersolvable for each $p$ dividing $o(A)$, where $J_{p}$ denotes the cyclic group of order $p$.

Proof. If $A$ is not nilpotent, $W$ is not supersolvable by Lemma 1 , while if $p \mid o(A)$ and $J_{p}$ wr $B$ is not supersolvable, then $A$ wr $B$ is not supersolvable by $(0.2)$ and $(0.7)$.

For sufficiency, induct on $o(A)$. There is a subgroup $H$ of $Z(A)$ of prime order. Since $H$ wr $B$ is supersolvable, there is an invariant series of $W$ up through $H^{B}$ with factors of prime order. Then $W / H \cong(A / H)$ wr $B$ is supersolvable by induction, and therefore $W$ is also supersolvable.

LemMa 4. If $p \nmid n, p$ prime, then $J_{p} \mathrm{wr} J_{n}$ is supersolvable if and only if $p \equiv 1(\bmod n)$.

Proof. Since $J_{n}$ is supersolvable and $W / F \cong J_{n}$, and $F$ is Abelian, it is necessary and sufficient to show that $F$ has a series $F=F_{n}$, $F_{n-1}, \cdots, F_{0}=E$ with each $o\left(F_{i+1} / F_{i}\right)=p$ and each $F_{i}$ invariant under the action of $B=J_{n}$. Here we may view $F$ as a vector space of dimension $n$ over $G F(p)$, a field with $p$ elements, acted on by the cyclic linear transformation $b$ induced by $b$, where $\langle b\rangle=B=J_{n}$. The minimum polynomial of $b$ is $\lambda^{n}-1$, and the subspaces invariant under $b$ may be put in one-one correspondence with the factors of $\lambda^{n}-1$ which have leading coefficient 1 , with one such subspace containing another if and only if the factor corresponding to the first divides the factor corresponding to the second [4, p. 129]. Therefore $J_{p}$ wr $J_{n}$ is supersolvable if and only if $\lambda^{n}-1$ splits into linear factors over $G F(p)$. Since $p \nmid n, \lambda^{n}-1$ has no repeated roots in $G F(p)$, and so $\lambda^{n}-1$ splits into linear factors if and only if $n \mid(p-1)$, for the roots of $\lambda^{n}-1$ form a subgroup of the multiplicative group of $G F(p)$.

Lemma 5. If $B$ is Abelian and $p \nmid o(B)$, then $J_{p}$ wr $B$ is supersolvable if and only if $p \equiv 1(\bmod m)$, where $m=\operatorname{Exp} B$.

Proof. If $p \not \equiv 1(\bmod m)$, we may apply $(0.2),(0.7)$, and Lemma 4 to conclude that $J_{p}$ wr $B$ is not supersolvable since $J_{p}$ wr $J_{m}$ is not. As in the proof of Lemma 4 , it is now sufficient to show that if $p \equiv 1$ $(\bmod m)$ then $F$ has a series $F=F_{n}, F_{n-1}, \cdots, F_{0}=E$ with each $o\left(F_{i+1} / F_{i}\right)=p$ and each $F_{i}$ invariant under the action of $B$, where in the present case $n=o(B)$. We interpret $B$ as a group acting on $F$, a vector space of dimension $n$ over $G F(p)$, and apply Maschke's 
Theorem [2, pp. 40-41] to conclude that $F$ is a direct sum of irreducible $B$-subspaces. If all of these irreducible $B$-subspaces are of dimension one, it is clear how to construct the sequence $F=F_{n}$, $F_{n-1}, \cdots, F_{0}=E$. However, since $p \nmid \operatorname{Exp} B$, it follows [2, Theorem 9.10, p. 37] that $B$ has $o(B)$ distinct one-dimensional $G F(p)$-representations, and therefore all irreducible $B$-subspaces are one-dimensional $\left[2\right.$, p. 213]. (Since $p \equiv 1(\bmod m), \lambda^{m}-1$ splits into linear factors over $G F(p)$, and so in the notation of $[2], \sqrt[m]{ } 1 \in G F(p)$.)

Lemma 6. If $P$ is a Sylow p-subgroup of $B$ containing $B^{\prime}$, then $J_{p}$ wr $B$ is supersolvable if and only if $p \equiv 1(\bmod m)$, where $m=\operatorname{Exp}(B / P)$.

Proof. Suppose $J_{p}$ wr $B$ is supersolvable. Since $P \triangleleft B, P$ has a complement $Q$ in $B$ by Schur's splitting theorem $[8,9.3 .6]$. Then $J_{p}$ wr $Q$ is supersolvable by $(0.2)$ and $(0.7)$, and hence $p \equiv 1(\bmod m)$ by Lemma 5 . Now assume $p \equiv 1(\bmod m)$. We can use the fact that $P \triangleleft B$ to conclude that $F P \triangleleft W$, and thereby think of $W$ as an extension of $F P$ by $B / P$. Then Lemmas 3 and 5 and (0.3) and (0.7) apply to yield that $J_{p}$ wr $B$ is supersolvable.

Proof of Theorem. If $A$ wr $B$ is supersolvable, the necessity of (i) follows from Lemma 1, that of (ii) from Lemma 2, and that of (iii) from Lemma 3 and Lemma 6 . Sufficiency is shown by Lemmas 3, 5, 6 , and (0.4).

Proof of Corollary. Apply the Theorem, (0.3), and (0.7).

The author is grateful to the referee for correcting an error in the original version of Lemma 6.

\section{REFERENCES}

1. G. Baumslag, Wreath products and p-groups, Proc. Cambridge Philos. Soc. 55 (1959), 224-231.

2. C. Curtis and I. Reiner, Representation theory of finite groups and associative algebras, Interscience, New York, 1962.

3. M. Hall, The theory of groups, Macmillan, New York, 1959.

4. N. Jacobson, Lectures on abstract algebra, Vol. II, Van Nostrand, New York, 1952.

5. L. Kaloujnine and M. Krasner, Produit complet des groupes de permutations et le problème d'extension de groupes. III, Acta. Sci. Math. Szeged 14 (1951), 69-82.

6. P. M. Neumann, On the structure of standard wreath products of groups, Math. Z. 84 (1964), 343-373.

7. B. H. Neumann, Hanna Neumann and P. M. Neumann, Wreath products and varieties of groups, Math. Z. 80 (1962), 44-62.

8. W. R. Scott, Group theory, Prentice-Hall, Englewood Cliffs, N. J., 1964.

The UNIVERSity OF TEXAS 